\title{
Per Low Powered Field
}

National Cancer Institute

\section{Source}

National Cancer Institute. Per Low Powered Field. NCI Thesaurus. Code C96620.

A unit of measure equal to the instances of an entity per visual field of a microscope set

to its minimum magnification power. 